\title{
Clinical efficacy of erlotinib, a salvage treatment for non-small cell lung cancer patients following gefitinib failure
}

Kyoung Min Cho ${ }^{1,2}$, Bhumsuk Keam ${ }^{1,3}$, Tae Min Kim, ${ }^{1,3}$ Se-Hoon Lee ${ }^{1,3}$, Dong-Wan Kim²,3 and Dae Seog Heo ${ }^{1,3}$

${ }^{1}$ Department of Internal Medicine, Seoul National University Hospital, Seoul; ${ }^{2}$ Department of Internal Medicine, Kyung Hee University School of Medicine, Seoul; ${ }^{3}$ Cancer Research Institute, Seoul National University College of Medicine, Seoul, Korea

Received: August 26, 2014 Revised : September 29, 2014 Accepted: December 5, 2014

\section{Correspondence to}

Bhumsuk Keam, M.D.

Department of Internal Medicine, Seoul National University Hospital, 101 Daehak-ro, Jongno-gu, Seoul 03080, Korea

Tel: +82-2-2072-7215

Fax: +82-2-762-9662

E-mail:bhumsuk@snu.ac.kr
Background/Aims: The purpose of this study was to identify predictive factors for erlotinib treatment in non-small cell lung cancer (NSCLC) patients following gefitinib failure.

Methods: Forty-five patients with NSCLC who were treated with erlotinib following gefitinib failure at Seoul National University Hospital between August 2005 and November 2011 were enrolled. Epidermal growth factor receptor (EGFR) mutation status, pathologic findings and other clinical factors, including response to tyrosine kinase inhibitors (TKIs) and progression-free survival (PFS), were evaluated.

Results: Of the 45 patients, 40 patients (88.8\%) had adenocarcinoma. The following EGFR mutations were observed: five patients with a deletion of exon 19, six patients with an L858R mutation, three patients with wild-type EGFR, and 31 patients with unknown mutations. The response rate of erlotinib was $4.4 \%$, and stable disease was $42.2 \%$. The median PFS for erlotinib was 2.6 months $(95 \%$ confidence interval, 1.4 to 3.7). Patients with a PFS $\geq 4$ months during previous gefitinib treatment had a significantly longer PFS with erlotinib (3.3 months vs. 1.6 months, respectively; $p<0.01$ ) than patients with PFS $<4$ months with gefitinib. According to multivariate analyses, $\mathrm{PFS} \geq 4$ months for previous gefitinib treatment was significantly associated with prolonged PFS with erlotinib $(p=0.04)$. However, the response rate of gefitinib and treatment sequence were not associated with prolonged PFS with erlotinib ( $p=0.28$ and $p=0.67$, respectively).

Conclusions: Following rechallenge with the EGFR TKI erlotinib following gefitinib failure, patients who showed prolonged PFS with gefitinib benefit from erlotinib. However, further prospective studies are needed to confirm these findings.

Keywords: Gefitinib; Erlotinib; Carcinoma, non-small-cell lung; Prognostic factor; Disease-free survival

\section{INTRODUCTION}

Epidermal growth factor receptor (EGFR) tyrosine kinase inhibitors (TKIs), which block the intracellular tyrosine domain of the receptor, inhibit tumor cell proliferation, invasion, angiogenesis, metastasis, and apoptosis in non-small cell lung cancer (NSCLC) [1,2]. Gefitinib is widely used for NSCLC patients with EGFR mutations. As a first-line treatment, gefitinib exhibits a median progression-free survival (PFS) of 9 to 13 months 
and a median overall survival (OS) of 19 to 31 months $[3,4]$. However, all patients eventually became resistant to gefitinib and experience progressive disease. Following gefitinib failure, most patients receive chemotherapy. However, it was recently suggested that rechallenging with an EGFR TKI after gefitinib may be effective [5-8].

Gefitinib and erlotinib have different pharmacological properties. Gefitinib is more susceptible to cytochrome-mediated metabolism, which may contribute to increased gefitinib clearance and lower systemic exposure [9]. The maximum tolerated dose of erlotinib is $150 \mathrm{mg}$, whereas the maximum tolerated dose of gefitinib is $250 \mathrm{mg}$ [10]. It has been suggested that erlotinib has a several-times greater area under the curve than gefitinib, which may explain its better clinical activity [11]. Moreover, erlotinib may be used following gefitinib failure. Studies have reported that erlotinib is effective when gefitinib treatment fails [5,12-14]. However, gefitinib is ineffective when erlotinib fails [15].

According to previous reports of salvage erlotinib following gefitinib failure, the PFS of erlotinib was 1.7 to 4.0 months $[5,6]$, and the disease control rate (DCR) was 29.2\% [8]. However, most reports examined a relatively small number of patients, case reports or pooled analyses of previous data [5-8]. Furthermore, there are limited data regarding other predictive factors for erlotinib following gefitinib failure.

In this study, we aimed to identify other predictive factors that impact the response of erlotinib following gefitinib failure in patients with NSCLC.

\section{METHODS}

\section{Patients and treatment}

We retrospectively reviewed the medical records of 45 patients with NSCLC who were treated with erlotinib following failure of gefitinib at Seoul National University Hospital between August 2005 and November 2011. Among the 45 patients in our hospital study, 12 had also been included in a previously published study concerning "erlotinib after gefitinib failure" [6].

The daily dose of gefitinib was $250 \mathrm{mg}$ until disease progression. Tumor response was assessed using the Response Evaluation Criteria in Solid Tumors (RECIST 1.1) [16]. Progression of gefitinib was confirmed using
RECIST 1.1 criteria. After gefitinib failure, patients received either erlotinib or cytotoxic chemotherapy followed by erlotinib. The daily dose of erlotinib was 150 mg until progression.

This study was approved by the Institutional Review Board of Seoul National University Hospital (approval number: $\mathrm{H}-1403-080-564)$. We also adhered to the Declaration of Helsinki regarding biomedical research involving human subjects.

\section{Mutational analyses}

EGFR mutational status was analyzed as previously described [17]. Briefly, tumor genomic DNA was extracted from five $5-\mu \mathrm{m}$ paraffin sections of each tumor block. Coding sequences from exons 18 to 21 were amplified by polymerase chain reaction using sequence-specific forward and reverse primers.

\section{Statistical analyses}

Statistical analyses of categorical variables were performed using Pearson chi-square tests. OS was calculated from the date of first treatment with erlotinib until the date of death. PFS was calculated from the date of first treatment with gefitinib or erlotinib until the date of progression or death. The median duration of PFS and OS was calculated using the Kaplan-Meier method. Survival comparisons between groups were performed using the log-rank test. The Cox proportional hazards regression model was used for multivariate analyses to assess the effect of patient characteristics and other prognostic factors. Two-sided $p$ values less than 0.05 were considered statistically significant. All analyses were performed using SPSS version 19 (IBM Co., Armonk, NY, USA).

\section{RESULTS}

\section{Study population and treatment patterns}

Baseline characteristics of the 45 patients are shown in Table 1. Female patients (73.3\%), non-smokers (77.8\%), and adenocarcinoma (88.8\%) were predominant in our study population. Results of the EGFR mutational analyses were as follows: five patients with a deletion of exon 19, six patients with an L858R mutation, three patients with wild-type EGFR, and 31 patients with unknown mu- 
Table 1. Patient baseline characteristics

\begin{tabular}{|c|c|}
\hline Characteristic & Value \\
\hline Age, yr, median (range) & $52(33-85)$ \\
\hline \multicolumn{2}{|l|}{ Smoking status } \\
\hline Current or former smoker & $9(20.0)$ \\
\hline Non-smoker & $35(77.8)$ \\
\hline Not available & $1(2.2)$ \\
\hline \multicolumn{2}{|l|}{ Sex } \\
\hline Female & $33(73 \cdot 3)$ \\
\hline Male & $12(26.7)$ \\
\hline \multicolumn{2}{|l|}{ Pathology } \\
\hline Adenocarcinoma & $40(88.8)$ \\
\hline Bronchoalveolar carcinoma & $2(4 \cdot 4)$ \\
\hline Non-small cell carcinoma, not otherwise specified & $2(4.4)$ \\
\hline Squamous cell carcinoma & $1(2.2)$ \\
\hline \multicolumn{2}{|l|}{ ECOG performance status } \\
\hline $0-1$ & $10(22.2)$ \\
\hline$\geq 2$ & $14(31.0)$ \\
\hline Not available & $21(46.6)$ \\
\hline \multicolumn{2}{|l|}{ EGFR mutation } \\
\hline Exon 19 microdeletion & $5(11.1)$ \\
\hline L858R in exon 21 & $6(13 \cdot 3)$ \\
\hline Wild-type & $3(6.7)$ \\
\hline Unknown & $31(68.9)$ \\
\hline \multicolumn{2}{|l|}{ Erlotinib as a salvage treatment sequence } \\
\hline Gefitinib $\rightarrow$ erlotinib & $25(55 \cdot 6)$ \\
\hline Gefitinib $\rightarrow$ cytotoxic chemotherapy $\rightarrow$ erlotinib & $20(44.4)$ \\
\hline Time between final gefitinib treatment to first erlotinib treatment, mon & $p<0.01$ \\
\hline Gefitinib $\rightarrow$ erlotinib & $0.8(0.2-1.4)$ \\
\hline Gefitinib $\rightarrow$ cytotoxic chemotherapy $\rightarrow$ erlotinib & $4.7(4.0-5 \cdot 4)$ \\
\hline Objective response rate of gefitinib & $26(57.8)$ \\
\hline Complete response & o \\
\hline Partial response & $26(57.8)$ \\
\hline Stable disease & $13(28.9)$ \\
\hline Progressive disease & $6(13 \cdot 3)$ \\
\hline Progression-free survival of gefitinib, mon & $6.8(3.9-9.6)$ \\
\hline \multicolumn{2}{|l|}{ Chemotherapy line of erlotinib } \\
\hline 2nd line chemotherapy & $6(13 \cdot 3)$ \\
\hline 3rd line chemotherapy & $13(28.9)$ \\
\hline$\geq 4^{\text {th }}$ line chemotherapy & $26(57 \cdot 7)$ \\
\hline \multicolumn{2}{|l|}{ Erlotinib patients by gefitinib chemotherapy line } \\
\hline Gefitinib as 1st chemotherapy line & $14(31.1)$ \\
\hline Gefitinib as $\geq 2$ nd chemotherapy line & $31(68.9)$ \\
\hline PFS of erlotinib by gefitinib chemotherapy line, mon & $p=0.70$ \\
\hline Gefitinib as 1st chemotherapy line & $3(1.3-4.7)$ \\
\hline Gefitinib as $\geq 2$ nd chemotherapy line & $2.6(1.5-3.7)$ \\
\hline
\end{tabular}

Values are presented as number (\%) or median ( $95 \%$ confidence interval).

ECOG, Eastern Cooperative Oncology Group; EGFR, epidermal growth factor receptor. 
tations. Twenty-five patients $(55.6 \%)$ received erlotinib followed by gefitinib, and 20 patients $(44.4 \%)$ received erlotinib followed by subsequent intervening cytotoxic chemotherapy. The response rate (RR) of gefitinib was $57.8 \%$ (95\% confidence interval [CI], 43.3 to 72.2 ), and the PFS of gefitinib was 6.8 months (95\% CI, 5.0 to 8.6). Except for one patient who received erlotinib following gefitinib due to gefitinib liver toxicity, all patients received erlotinib treatment after gefitinib because of disease progression.

\section{Clinical efficacy of erlotinib following gefitinib failure}

The complete response (CR), partial response (PR), and

Table 2. Clinical efficacy of erlotinib following gefitinib failure

\begin{tabular}{lc}
\hline Clinical efficacy of erlotinib & Value \\
\hline Response of erlotinib & \\
\hline Complete response & 0 \\
\hline Partial response & $2(4.4)$ \\
Stable disease & $19(42.2)$ \\
Progressive disease & $24(53.3)$ \\
\hline PFS of erlotinib, mon & $2.6(1.4-3.7)$ \\
\hline OS $^{\text {a }}$ of erlotinib, mon & $8.0(4.4-11.6)$ \\
\hline
\end{tabular}

Values are presented as number (\%) or median (95\% confidence interval).

PFS, progression-free survival; OS, overall survival.

${ }^{\mathrm{a}} \mathrm{OS}$ calculated from the start date of erlotinib until death. stable disease (SD) of erlotinib were o\%, $4.4 \%$, and $42.2 \%$, respectively. The RR of erlotinib was $4.4 \%$ (95\% CI, o to 10.3), and the DCR was $46.6 \%$ (95\% CI, 32.1 to 61.2). The median PFS and OS for erlotinib were 2.6 months (95\% CI, 1.4 to 3.7 ) and 8.0 months (95\% CI, 4.4 to 11.6), respectively (Table 2). There were no differences between Eastern Cooperative Oncology Group performance score, RR of erlotinib, or DCR of erlotinib with PFS following prior gefitinib treatment ( $\mathrm{PFS}<4$ months vs. $\geq 4$ months) (Table 3).

\section{Predictive factors for the PFS of erlotinib}

There was no difference in the PFS of erlotinib based on treatment sequence. PFS values of erlotinib between patients treated with erlotinib followed by gefitinib and those treated with erlotinib followed by gefitinib and intervening chemotherapy were 3.3 and 2.2 months, respectively $(p=0.67$ ) (Fig. 1). There was no significant difference in the PFS of erlotinib with respect to previous gefitinib RR (CR + PR vs. PD, 3.3 and 2.2 months, respectively; $p=0.29$ ) (Fig. 2).

However, patients with PFS $\geq 4$ months with prior gefitinib treatment had significantly longer erlotinib PFS compared with those with previous gefitinib PFS $<4$ months ( 3.3 and 1.6 months, respectively; $p<0.01$ ) (Table 4, Fig. 3). Based on multivariate analyses, $\mathrm{PFS} \geq 4$ months with prior gefitinib treatment was a significant prognostic factor for erlotinib PFS (hazard ratio, 0.27 ; 95\% CI, 0.3 to $2.3 ; p=0.04)(4)$.

Table 3. Erlotinib response by progression-free survival of prior gefitinib

\begin{tabular}{|c|c|c|c|}
\hline \multirow{2}{*}{ Variable } & \multicolumn{2}{|c|}{ Previous gefitinib progression-free survival } & \multirow{2}{*}{$p$ value } \\
\hline & $<4$ months $(\mathrm{n}=10)$ & $\geq 4$ months $(\mathrm{n}=34)$ & \\
\hline ECOG performance status score & & & 1.00 \\
\hline $0-1$ & $2(40.0)$ & $8(42.1)$ & \\
\hline$\geq 2$ & $3(60.0)$ & $11(57.9)$ & \\
\hline Response rate of erlotinib & & & 1.00 \\
\hline $\mathrm{CR}+\mathrm{PR}$ & o & $2(7.7)$ & \\
\hline $\mathrm{SD}+\mathrm{PD}$ & $8(100.0)$ & $24(92.3)$ & \\
\hline Disease control rate of erlotinib & & & 0.11 \\
\hline $\mathrm{CR}+\mathrm{PR}+\mathrm{SD}$ & $2(25.0)$ & $16(61.5)$ & \\
\hline PD & $6(75.0)$ & $10(38.5)$ & \\
\hline
\end{tabular}

Values are presented as number (\%). One patient did not have the progression-free survival of erlotinib evaluated because erlotinib was stopped early due to liver toxicity.

ECOG, Eastern Cooperative Oncology Group; CR, complete response; PR, partial response; SD, stable disease; PD, progressive disease. 


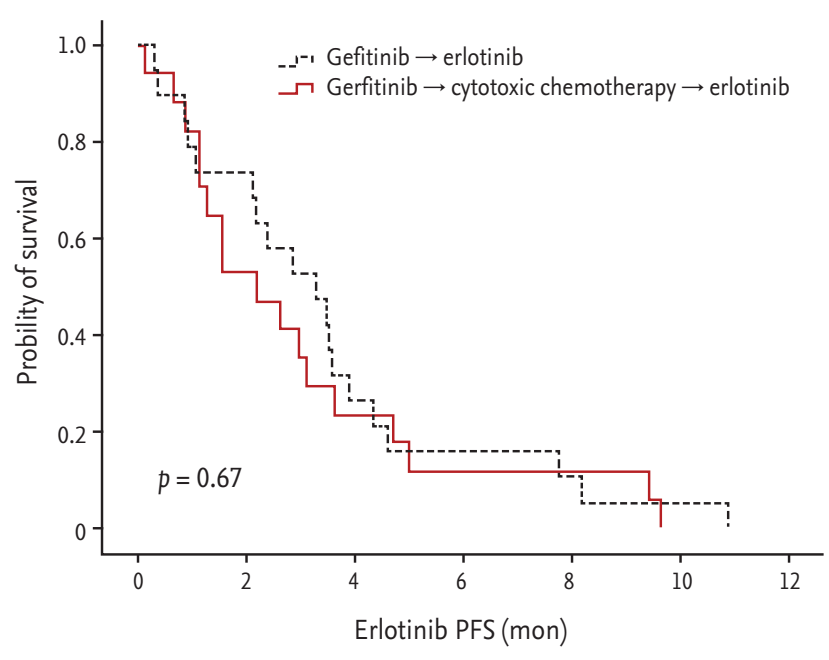

Figure 1. Progression-free survival (PFS) of erlotinib by treatment sequence.

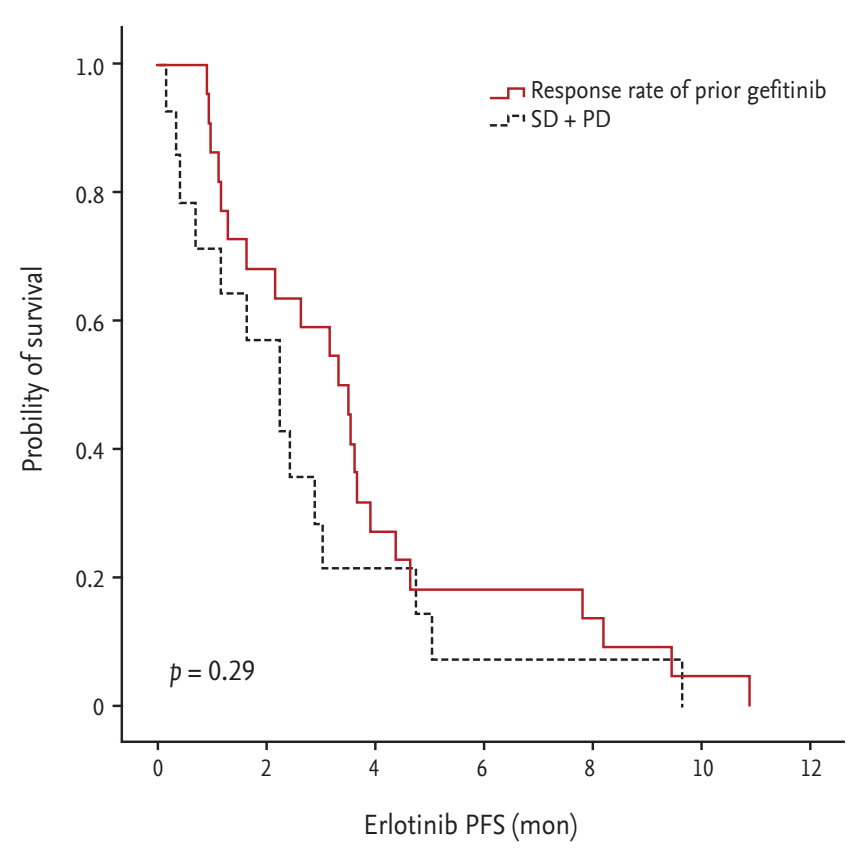

Figure 2. Progression-free survival (PFS) of erlotinib by the response rate of prior gefitinib. $\mathrm{SD}$, stable disease; $\mathrm{PD}$, progressive disease.

\section{DISCUSSION}

In the present study, we found that the PFS of prior gefitinib is an important predictive factor for the clinical efficacy of erlotinib following gefitinib failure. A PFS $\geq$ 4 months for prior gefitinib treatment was also significantly associated with prolonged PFS with erlotinib.

Kaira et al. [8] reported differential responses to er-

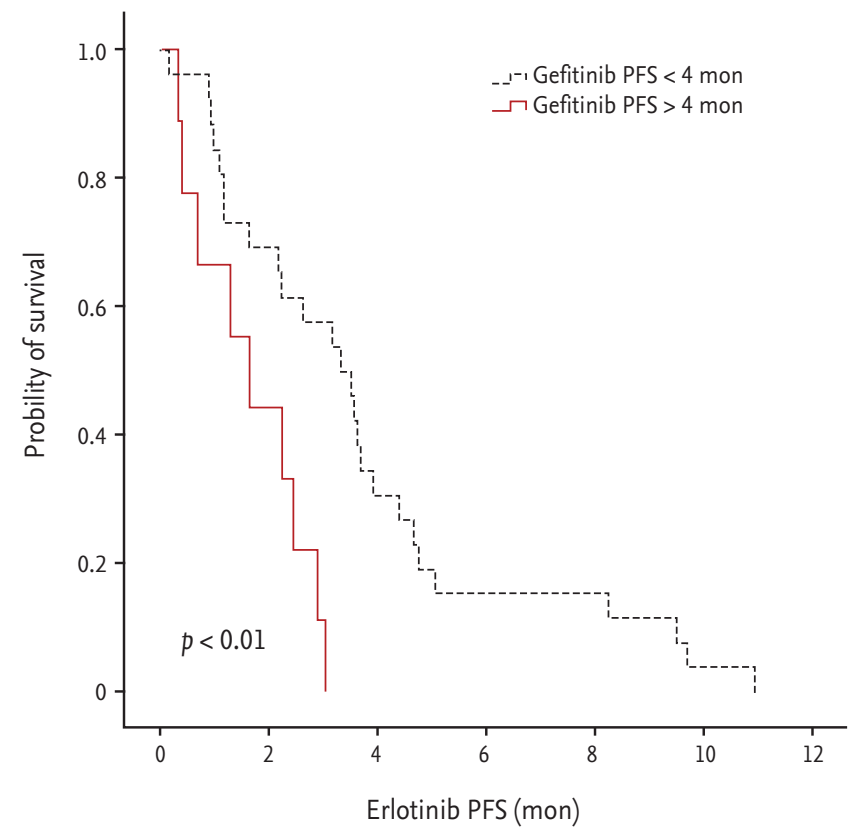

Figure 3. Progression-free survival (PFS) of erlotinib by the PFS of prior gefitinib.

lotinib therapy in patients that showed SD and PFS of longer than 6 months during gefitinib treatment. Cho et al. [5] reported that patients with SD while receiving gefitinib showed significantly higher DCR and RR during erlotinib treatment. In our study, erlotinib response (RR, DCR) was not associated with PFS (< 4 months vs. $\geq$ 4 months) of prior gefitinib treatment, and only PFS of erlotinib was influenced by PFS of prior gefitinib treatment. Data regarding EGFR TKI retreatment suggest that the duration of maintenance treatment with previous gefitinib is an important predictive factor of erlotinib treatment duration, rather than previous gefitinib response. This is similar to a report that found SD of previous gefitinib treatment is an important factor, thus implying that the duration of maintenance with a previous EGFR TKI is important [5].

In this study, the median OS and PFS of erlotinib were 8.0 and 2.6 months, respectively. These results agree with data from previous reports indicating that the median OS and PFS of EGFR TKI retreatment are 9.0 months and 1.7 to 4.0 months, respectively [5,18]. In addition to a median PFS of 9 to 13 months for first-line EGFR TKI therapy $[3,4]$ and a gefitinib PFS of 6.8 months, our study found four patients with an erlotinib PFS longer than 8 months with effective treatment duration. 
Table 4. Multivariate Cox proportional hazard regression analyses for the PFS of erlotinib

\begin{tabular}{|c|c|c|c|c|c|c|}
\hline \multirow{2}{*}{ Variable } & \multirow{2}{*}{ Median PFS, mon } & \multirow{2}{*}{$95 \% \mathrm{CI}$} & \multirow{2}{*}{$p$ value } & \multicolumn{3}{|c|}{ Multivariate analyses } \\
\hline & & & & HR & $95 \% \mathrm{CI}$ & $p$ value \\
\hline \multicolumn{7}{|l|}{ Age, yr } \\
\hline$<60$ & 2.6 & $1.6-3.6$ & 0.37 & 1 & & 0.67 \\
\hline$\geq 60$ & 2.2 & $0-4.8$ & & 0.67 & $0.4-2.0$ & \\
\hline \multicolumn{7}{|l|}{ Sex } \\
\hline Male & 2.6 & $1.6-3.6$ & 0.80 & 1 & & \\
\hline Female & 2.4 & $0.9-3.9$ & & 0.91 & $0.4-2.1$ & 0.91 \\
\hline \multicolumn{7}{|c|}{ PFS of prior gefitinib } \\
\hline $\mathrm{PFS}<4$ mon & 1.6 & $0.6-2.6$ & $<0.01$ & 1 & & \\
\hline $\mathrm{PFS} \geq 4$ mon & $3 \cdot 3$ & $2.1-4.5$ & & 0.27 & $0.3-2.3$ & 0.04 \\
\hline \multicolumn{7}{|c|}{ Gefitinib objective response } \\
\hline $\mathrm{PR}+\mathrm{CR}$ & $3 \cdot 3$ & $2.3-4.4$ & 0.29 & 1 & & \\
\hline $\mathrm{PD}$ & 2.2 & $1.1-3 \cdot 3$ & & 0.87 & $0.3-2.3$ & 0.79 \\
\hline
\end{tabular}

PFS, progression-free survival; CI, confidence interval; HR, hazard ratio; PR, partial response; CR, complete response; PD, progressive disease.

Our study also found that a shorter PFS ( $>4$ months) can be expected for efficacy with EGFR TKI retreatment compared with previous data (PFS $>6$ months). If the median PFS of prior gefitinib is longer than 4 months, erlotinib may be considered a salvage treatment. Furthermore, four male patients who smoked with adenocarcinoma histology showed $>3$ months of erlotinib PFS (9.7, 3.1, 8.2, and 4.6 months, respectively). Thus, salvage erlotinib following gefitinib failure can be used in patients with unfavorable clinical factors for TKIs. These results are in agreement with those of a previous report [6]. Although our study included 12 patients from a previous report [6], considering that all patients in previous reports were female non-smokers, our results provide additional insights into the efficacy of erlotinib following gefitinib failure.

Based on our results, we believe there may be an unknown mechanism, EGFR TKI sensitivity, or resistance genes associated with previous treatment duration and retreatment duration. Wong et al. [13] suggested the possibility of an unknown non-cross sensitivity or resistance differential mutation. Choong et al. [19] reported the novel discovery of an L858R + E884K somatic mutation in exon [20], suggesting differential sensitivity of gefitinib and erlotinib [19]. Further gene mutation analyses may clarify the mechanism of erlotinib effectiveness following gefitinib failure.
EGFR TKI mutations consist of the T790M point mutation in exon 20 of EGFR (50\%), amplification of the MET gene (20\%), and other mutations (30\%) in resistance cases [21-24]. A patient who developed resistance to gefitinib had one tumor with a T790M mutation in EGFR and a MET amplification in the other, indicative of heterogeneous EGFR resistance distribution [23]. The tumor mass comprises both EGFRTKI-sensitive and -resistant clones, regardless of the molecular mechanism. EGFR TKI treatment continuously decreases drug-sensitive clones and increases EGFR TKI-resistant clones [25]. Retreatment efficacy may be affected by a drug holiday, which allows the formation of EGFR TKI-sensitive clones [26]. Guo et al. [27] suggested that chemotherapy may modify EGFR-sensitive cells dominated by an unknown mechanism, which would kill EGFR-resistant cells and induce other genetic mutations that regulate EGFR-resistant clones. Other reports have supported these findings $[18,28,29]$. According to another report, intervening cytotoxic chemotherapy between gefitinib and erlotinib improved the efficacy of salvage erlotinib treatment [30]. Taken together, our study shows a significantly different meaningful duration between final gefitinib treatment and first erlotinib treatment $(p<$ o.01). Additionally, our study shows that chemotherapy following gefitinib failure may not affect the PFS of erlotinib. Thus, it was not shown whether the duration 
between EGFR TKI therapy and EGFR TKI rechallenge may affect the response, indicating that the regrowth of clones sensitive to an EGFR TKIs can respond again to a rechallenge with an EGFR TKI.

Our study has several limitations. First, our study is retrospective in nature. Second, most patients (68.9\%) did not undergo EGFR mutational analyses. However, recent studies have shown no difference in the PFS of erlotinib following gefitinib failure when evaluating wild-type EGFR and EGFR mutations [8,31]. Cho et al. [5] reported erlotinib efficacy in patients with wild-type EGFR following gefitinib failure. Further investigations are warranted to determine whether differences in erlotinib PFS correlate with EGFR TKI resistance mutations (e.g., T790M point mutation, MET gene amplification, or K-ras mutation). However, the current study was conducted on a larger scale than that previously reported $[5-7,13,32,33]$, and demonstrated the efficacy of salvage erlotinib in cases with unsatisfactory clinical factors for TKIs.

In conclusion, when rechallenging with the EGFR TKI erlotinib following gefitinib failure, patients who showed prolonged PFS with gefitinib may benefit from erlotinib treatment. Further prospective studies and confirmation are warranted.

\section{KEY MESSAGE}

1. Following rechallenge with the epidermal growth factor receptor tyrosine kinase inhibitors erlotinib following gefitinib failure, patients who showed prolonged progression-free survival with gefitinib benefit from erlotinib.

\section{Conflict of interest}

No potential conflict of interest relevant to this article was reported.

\section{Acknowledgments}

This study was supported by grants from the Innovative Research Institute for Cell Therapy, Republic of Korea (Ao62260). This work was also supported by a National Research Foundation of Korea (NRF) grant funded by the Korean Government (2010-0009563).

\section{REFERENCES}

1. Janne PA, Engelman JA, Johnson BE. Epidermal growth factor receptor mutations in non-small-cell lung cancer: implications for treatment and tumor biology. J Clin Oncol 2005;23:3227-3234.

2. Baselga J, Arteaga CL. Critical update and emerging trends in epidermal growth factor receptor targeting in cancer. J Clin Oncol 2005;23:2445-2459.

3. Maemondo M, Inoue A, Kobayashi K, et al. Gefitinib or chemotherapy for non-small-cell lung cancer with mutated EGFR. N Engl J Med 2010;362:2380-2388.

4. Mitsudomi T, Morita S, Yatabe Y, et al. Gefitinib versus cisplatin plus docetaxel in patients with non-small-cell lung cancer harbouring mutations of the epidermal growth factor receptor (WJTOG3405): an open label, randomised phase 3 trial. Lancet Oncol 2010;11:121-128.

5. Cho BC, Im CK, Park MS, et al. Phase II study of erlotinib in advanced non-small-cell lung cancer after failure of gefitinib. J Clin Oncol 2007;25:2528-2533.

6. Sim SH, Han SW, Oh DY, et al. Erlotinib after Gefitinib failure in female never-smoker Asian patients with pulmonary adenocarcinoma. Lung Cancer 2009;65:204-207.

7. Viswanathan A, Pillot G, Govindan R. Lack of response to erlotinib after progression on gefitinib in patients with advanced non-small cell lung cancer. Lung Cancer 2005:50:417-418.

8. Kaira K, Naito T, Takahashi T, et al. Pooled analysis of the reports of erlotinib after failure of gefitinib for non-small cell lung cancer. Lung Cancer 2010;68:99-104.

9. Li J, Zhao M, He P, Hidalgo M, Baker SD. Differential metabolism of gefitinib and erlotinib by human cytochrome P450 enzymes. Clin Cancer Res 2007;13:3731-3737.

10. Comis RL. The current situation: erlotinib (Tarceva) and gefitinib (Iressa) in non-small cell lung cancer. Oncologist 2005;10:467-470.

11. Leveque D. Pharmacokinetics of gefitinib and erlotinib. Lancet Oncol 2011;12:1093.

12. Lee DH, Kim SW, Suh C, Yoon DH, Yi EJ, Lee JS. Phase II study of erlotinib as a salvage treatment for non-smallcell lung cancer patients after failure of gefitinib treatment. Ann Oncol 2008;19:2039-2042.

13. Wong AS, Soong R, Seah SB, et al. Evidence for disease control with erlotinib after gefitinib failure in typical gefitinib-sensitive Asian patients with non-small cell lung cancer. J Thorac Oncol 2008;3:400-404. 
14. Costa DB, Nguyen KS, Cho BC, et al. Effects of erlotinib in EGFR mutated non-small cell lung cancers with resistance to gefitinib. Clin Cancer Res 2008;14:7060-7067.

15. Grossi F, Rijavec E, Dal Bello MG, et al. The administration of gefitinib in patients with advanced non-small-cell lung cancer after the failure of erlotinib. Cancer Chemother Pharmacol 2012;69:1407-1412.

16. Eisenhauer EA, Therasse P, Bogaerts J, et al. New response evaluation criteria in solid tumours: revised RECIST guideline (version 1.1). Eur J Cancer 2009;45:228-247.

17. Kim YT, Kim TY, Lee DS, et al. Molecular changes of epidermal growth factor receptor (EGFR) and KRAS and their impact on the clinical outcomes in surgically resected adenocarcinoma of the lung. Lung Cancer 2008;59:111118.

18. Tomizawa Y, Fujita Y, Tamura A, et al. Effect of gefitinib re-challenge to initial gefitinib responder with non-small cell lung cancer followed by chemotherapy. Lung Cancer 2010;68:269-272.

19. Choong NW, Dietrich S, Seiwert TY, et al. Gefitinib response of erlotinib-refractory lung cancer involving meninges: role of EGFR mutation. Nat Clin Pract Oncol 2006;3:50-57.

20. Zhang Z, Lee JC, Lin L, et al. Activation of the AXL kinase causes resistance to EGFR-targeted therapy in lung cancer. Nat Genet 2012;44:852-860.

21. Kobayashi S, Boggon TJ, Dayaram T, et al. EGFR mutation and resistance of non-small-cell lung cancer to gefitinib. N Engl J Med 2005;352:786-792.

22. Pao W, Miller VA, Politi KA, et al. Acquired resistance of lung adenocarcinomas to gefitinib or erlotinib is associated with a second mutation in the EGFR kinase domain. PLoS Med 2005;2:e73.

23. Engelman JA, Zejnullahu K, Mitsudomi T, et al. MET amplification leads to gefitinib resistance in lung cancer by activating ERBB3 signaling. Science 2007;316:1039-1043.

24. Yano S, Wang W, Li Q, et al. Hepatocyte growth factor in- duces gefitinib resistance of lung adenocarcinoma with epidermal growth factor receptor-activating mutations. Cancer Res 2008;68:9479-9487.

25. Jang SH. Long term therapeutic plan for patients with non-small cell lung cancer harboring EGFR mutation. Tuberc Respir Dis (Seoul) 2014;76:8-14.

26. Becker A, Crombag L, Heideman DA, et al. Retreatment with erlotinib: regain of TKI sensitivity following a drug holiday for patients with NSCLC who initially responded to EGFR-TKI treatment. Eur J Cancer 2011;47:2603-2606.

27. Guo R, Chen X, Wang T, Zhang Z, Sun J, Shu Y. Subsequent chemotherapy reverses acquired tyrosine kinase inhibitor resistance and restores response to tyrosine kinase inhibitor in advanced non-small-cell lung cancer. BMC Cancer 2011;11:90.

28. Li XD, Geng YT, Wu CP, et al. Restoration of gefitinib efficacy following chemotherapy in a patient with metastatic non-small cell lung cancer. Onkologie 2010;33:466-469.

29. Yoshimoto A, Inuzuka K, Kita T, Kawashima A, Kasahara K. Remarkable effect of gefitinib retreatment in a patient with nonsmall cell lung cancer who had a complete response to initial gefitinib. Am J Med Sci 2007;333:221-225.

30. Hata A, Katakami N, Yoshioka H, et al. Erlotinib after gefitinib failure in relapsed non-small cell lung cancer: clinical benefit with optimal patient selection. Lung Cancer 2011;74:268-273.

31. Koyama N, Uchida Y. Clinical significance of erlotinib monotherapy for gefitinib-resistant non-small cell lung cancer with EGFR mutations. Anticancer Res 2013;33:50835089.

32. Vasile E, Tibaldi C, Chella A, Falcone A. Erlotinib after failure of gefitinib in patients with advanced non-small cell lung cancer previously responding to gefitinib. J Thorac Oncol 2008;3:912-914.

33. Sharma SV, Bell DW, Settleman J, Haber DA. Epidermal growth factor receptor mutations in lung cancer. Nat Rev Cancer 2007;7:169-181. 